\title{
Fresnel Diffraction Mirror for an Atomic Wave
}

\author{
Hilmar Oberst, ${ }^{1}$ Dimitrii Kouznetsov, ${ }^{1}$ Kazuko Shimizu, ${ }^{2}$ Jun-ichi Fujita, ${ }^{3}$ and Fujio Shimizu ${ }^{1,4}$ \\ ${ }^{1}$ Institute for Laser Science, University of Electro-Communications, Chofu, Tokyo, 182-8585, Japan \\ ${ }^{2}$ Department of Applied Physics and Chemistry, University of Electro-Communications, Chofu, Tokyo, 182-8585, Japan \\ ${ }^{3}$ University of Tsukuba, 1-1-1 Ten-nodai, Tsukuba, Ibaraki 305-8573, Japan \\ ${ }^{4}$ NTT Basic Research Laboratories, NTT Corporation, and CREST, Morinoshita-Wakamiya, Atsugi, 243-0198, Japan
}

(Received 6 September 2004; published 11 January 2005)

\begin{abstract}
We have experimentally demonstrated a material-independent mirror for atomic waves that uses the Fresnel diffraction at an array of parallel ridges. $\mathrm{He}^{*}\left(2{ }^{3} S_{1}\right)$ and $\mathrm{Ne}^{*}\left(1 s_{3}\right)$ atomic waves were reflected coherently on a silicon plate with a microfabricated grating structure, consisting of narrow wall-like ridges. We measured the reflectivity at grazing incidence as a function of the incident velocity and angle. Our data show that the reflectivity on this type of mirror depends only on the distance between the ridges, the wavelength, and the incident angle, but is insensitive to the material of the grating structure. The reflectivity is observed to increase by 2 orders of magnitude, compared to that of a flat polished silicon surface, where the reflection is caused by the attractive surface potential. For He* atoms, the measured reflectivity exceeds $10 \%$ for normal incident velocities below about $25 \mathrm{~cm} / \mathrm{s}$.
\end{abstract}

DOI: 10.1103/PhysRevLett.94.013203

PACS numbers: 34.50.Dy, 03.75.-b, 42.25.Fx

Optics has benefited from solid lenses and mirrors that use a surface polished to an accuracy much better than the optical wavelength. Such high quality optical components made it possible to construct a variety of precision optical devices that control the optical wave to diffraction-limited accuracy. Matter waves did not have such precise components mainly because the de Broglie wavelength at room temperature is much shorter than the length that characterizes the best prepared solid surfaces. As a result the wave front of the reflected matter wave is distorted, and the reflected beam has never been diffraction limited.

Recently, one of the authors has shown that a very cold atomic wave can be reflected coherently from a solid surface [1]. Diffraction-limited reflection can be observed because the de Broglie wavelength normal to the surface is large compared to the surface roughness in this case, and because atoms are reflected at some distance away from the real surface, where the roughness of the surface is averaged out. The cold atomic wave feels the attractive van der Waals potential near the solid surface sufficiently steep, to cause reflection by impedance mismatch of the wave vector. Such reflections have subsequently been studied with different solid surfaces and atomic species [2,3]. They have previously been known to occur when slow atoms are incident on liquid helium surfaces [4-6]. The theory of reflection at attractive potential wells has been extensively discussed in the literature [7-13].

Several other types of atom mirrors have been developed over the past decades, the most prominent being evanescent wave mirrors and mirrors using magnetic fields [1418]. These mirrors create artificial repulsive surface potentials that reflect the atoms very efficiently; however, it is quite difficult to create large mirrors that are sufficiently flat. In contrast, mirrors based on solid surfaces do not reflect atoms as efficiently, but they can be made very large and are inherently stable, accurate, and nearly dispersionless.

On a solid surface, the reflection due to the attractive van der Waals potential increases when the strength of the potential is reduced: a higher reflectivity is expected by reducing the density of the solid near the surface. Motivated by this consideration, two of the authors simulated a low density surface by forming the surface in a grating structure with narrow ridges and showed that the reflectivity increased dramatically [19]. Using this result they constructed a reflection-type amplitude hologram for atomic waves [20].

The dimension of the used grating structure, however, was orders of magnitude larger than the de Broglie wavelength of the incident atomic wave. The reflectivity was measured at the grazing angle, typically $10^{-2} \mathrm{rad}$ or less. In this experimental condition, the reflection is as well described by using a simple diffraction theory. We assume that one ridge of the grating is a thin knife edge whose face blocks the wave completely. The plane wave incident at a small angle $\theta$ hits the edge and is diffracted. The diffracted waves from all edges constructively add to form the specularly reflected beam. When we describe the reflection at the surface as the diffraction at an array of ridges, then we expect the reflectivity to depend mainly on the dimension of the structure, the wave vector, and the incident angle of the atomic wave, but the properties of the material should have little influence. This is, indeed, what we observe.

We can apply Fresnel-Kirchhoff's diffraction theory, when the spacing between the knife edges is much larger than the wavelength [21]. The reflectivity then becomes a function of only one parameter

$$
\beta=\sqrt{\frac{k L}{\pi}} \theta
$$


where $L$ is the spacing between the knife edges, $k$ is the wave vector, and $\theta$ the incident angle.

This Letter reports the first observation of the reflection from the Fresnel diffraction mirror for matter waves. We measured the reflectivity of a cold metastable helium and neon atomic wave on a silicon grating, which had wall-like ridges with a width approximately $100 \mathrm{~nm}$ and a periodicity of $5 \mu \mathrm{m}$. The reflectivity varies wildly with $\theta$, speed of the atom, and atomic species. However, we found that it depends mainly on $\beta$ of Eq. (1). This indicates that the influence of other parameters, such as the width of the ridges and the van der Waals interaction constant, is relatively small.

A numerical simulation of the diffraction and reflection of a plane wave at an array of idealized ridges has been done and will be published in a separate paper [22]. Here we derive the scaling parameter $\beta$ and the approximate dependence of the reflectivity $R(\beta)$. We consider an array of half planes that are placed perpendicular to the $x$ axis with the edge on the $y=0$ plane (see Fig. 1). A plane wave

$$
\psi(x, y)=\exp [i k x \cos (\theta)-i k y \sin (\theta)]
$$

is incident from the left and hits the first half plane at $x=$ 0 . The half plane blocks the wave in $y<0$ and produces a diffracted wave. Within the Fresnel diffraction approximation, and when $\theta \ll 1$, the amplitude at the next half plane at $x=L$ is

$$
\begin{aligned}
\psi(L, y) & =e^{i k\left(1-\theta^{2} / 2\right) L-i k \theta y}\left\{1-\frac{1}{1+i} E[\sqrt{k /(\pi L)}(y+L \theta)]\right\} \\
& \equiv e^{i k\left(1-\theta^{2} / 2\right) L-i k \theta y}-\psi_{s},
\end{aligned}
$$

where $E(u)=(1+i) / 2-\int_{0}^{u} \exp \left(-i \pi v^{2} / 2\right) d v$. The second term in the curly bracket, $\psi_{s}$, is the amplitude generated by the Fresnel diffraction. If this amplitude is much less than unity, we can neglect multiple scattering and assume that the diffracted wave at the next half plane, $x=$ $2 L$, is generated only by a plane wave whose amplitude is equal to that of the incident wave. Therefore, $\psi$ in Eq. (2) is the amplitude at every half plane, $x=n L$. The intensity of the reflected wave is obtained by integrating the contribution from all edges. Since the incoming wave is blocked at each edge in a region of width $L \theta$, the contribution to the

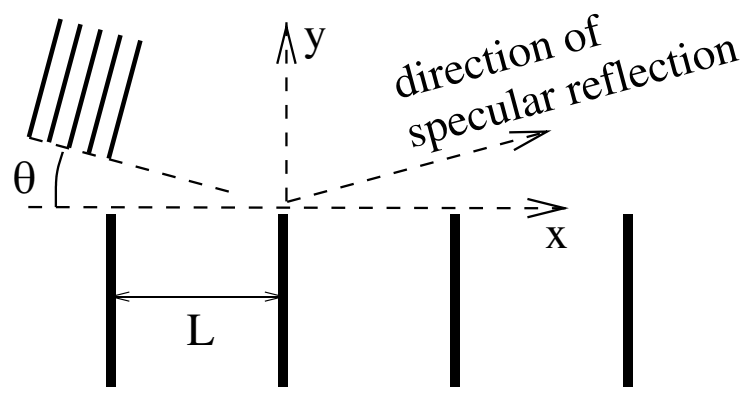

FIG. 1. A plane wave is scattered at an array of ridges. reflection per edge is approximately limited to the region between $y=0$ and $y=L \theta$. This contribution therefore is

$$
\phi_{r}=-\frac{1}{1+i} \int_{0}^{L \theta} e^{-2 i k \theta y} E[\sqrt{k /(\pi L)}(y+L \theta)] d y .
$$

The same quantity for the incident wave is $(L \theta)^{2}$, so we get the estimate for the reflectivity

$$
R(\beta)=\frac{\left|\phi_{r}\right|^{2}}{(L \theta)^{2}}=\frac{1}{2 \beta^{2}}\left|\int_{0}^{\beta} e^{-2 i \pi \beta t} E(t+\beta) d t\right|^{2} .
$$

This shows that the reflectivity depends only on the parameter $\beta$ defined in Eq. (1).

For an optical wave, the reflection from a structured surface has little practical use because a flat surface made of the same material always has higher reflectivity. For a matter wave, however, the reflectivity from a structured surface can be orders of magnitude higher than that from a flat surface, where the colliding particle feels a long range attractive potential.

The reflectivity was measured with metastable neon atoms in the $1 s_{3}$ state with the velocity approximately $3 \mathrm{~m} / \mathrm{s}$, and with metastable helium atoms in the $2^{3} S_{1}$ state at the velocity ranging from 30 to $130 \mathrm{~m} / \mathrm{s}$. The experimental setup for neon was described before in [19]. For $\mathrm{He}^{*}$ the method to generate the atomic beam is different from that for $\mathrm{Ne}^{*}$, because the optical pumping from a triplet state to a singlet state is practically forbidden, so that the triplet atoms must be released by using another method. The experimental setup used for He is schematically shown in Fig. 2. Metastable helium atoms in the $2^{3} S_{1}$ state are trapped and cooled in a magneto-optical trap (MOT) in a four-beam configuration. The MOT is loaded from an intense beam of $\mathrm{He}^{*}$, which is created in a liquid nitrogen-cooled dc discharge source, then collimated and slowed in a Zeeman decelerator. We use only the transition to the $2^{3} P_{2}$ state at $1083 \mathrm{~nm}$. The light source is a gratingstabilized diode laser followed by a Yb-doped fiber amplifier, and the laser frequency is stabilized using absorption spectroscopy in a helium discharge cell.

The He atoms are released from the trap by illuminating the cloud of trapped atoms from the top with short pulses of resonant light. The laser beam is focused to a $1 / e^{2}$ beam waist below $100 \mu \mathrm{m}$ providing an almost pointlike source of atoms. The released atoms fall along a vertical line and are partially reflected at the grazing angle on the silicon plate, placed $41 \mathrm{~cm}$ below the trap. The pattern of scattered atoms is detected with a gated microchannel plate (MCP) detector, placed $113 \mathrm{~cm}$ below the MOT. The detector provides a spatial resolution below $100 \mu \mathrm{m}$. We reduce the velocity spread to below $8 \%$ by detecting only atoms that arrive after a chosen delay and within a short interval. The length and intensity of the releasing laser pulses are adjusted to maximize the number of atoms within the chosen interval. The MOT is switched off during the detection interval. 
The pattern on the MCP detector consists of atoms passing behind the silicon plate, atoms passing in front of the silicon plate, and atoms reflected on the plate. The incident angle is changed by tilting the plate around a fixed axis at the top of the reflecting surface. A movable edge, placed $4 \mathrm{~cm}$ above the silicon plate, is used to block out directly falling atoms that would otherwise overlap with the reflected beam. The reflected atoms can be clearly distinguished from directly falling atoms down to incident angles of about $1 \mathrm{mrad}$. A HeNe laser beam is reflected on the silicon plate to obtain a scale for the angle between the surface and the atomic beam. The reflectivity is calculated as the ratio of the number of atoms in the reflected part and the number of incident atoms. The number of incident atoms has to be extrapolated: The number of atoms passing behind the plate is used to scale the intensity, and the effective mirror area is measured using the size of the projected shadow of the silicon plate on the detector.

The mirror we used was a $100 \times 20 \times 0.5 \mathrm{~mm}^{3}$ silicon plate with a microfabricated grating structure consisting of parallel narrow ridges with a period $L=5 \mu \mathrm{m}$. A scanning microscope photograph of the surface is shown in Fig. 3. The surface structure was formed using electron beam lithography, following a procedure similar to that described in [19]. The ridges had a nearly rectangular shape with a width at the top of $100 \mathrm{~nm}$. The top of the ridge was the (001) surface and was flat within $20 \mathrm{~nm}$.

The reflectivity measured on the silicon sample is shown in Fig. 4(a) as a function of the normal incident velocity. For $\mathrm{He}^{*}$, the parallel incident velocities were $v_{\|}=35,64$, 81 , and $128 \mathrm{~m} / \mathrm{s}$, and the incident angle was varied between 1 and $14 \mathrm{mrad}$. The reflectivity exceeds $10 \%$ for normal incident velocities below about $25 \mathrm{~cm} / \mathrm{s}$. For comparison we show the reflectivity of $\mathrm{He}^{*}$ on a flat polished $\mathrm{Si}$ surface measured in [23]. For $\mathrm{Ne}^{*}\left(1 s_{3},{ }^{3} P_{0}\right)$ the data have been measured on the same sample with the apparatus

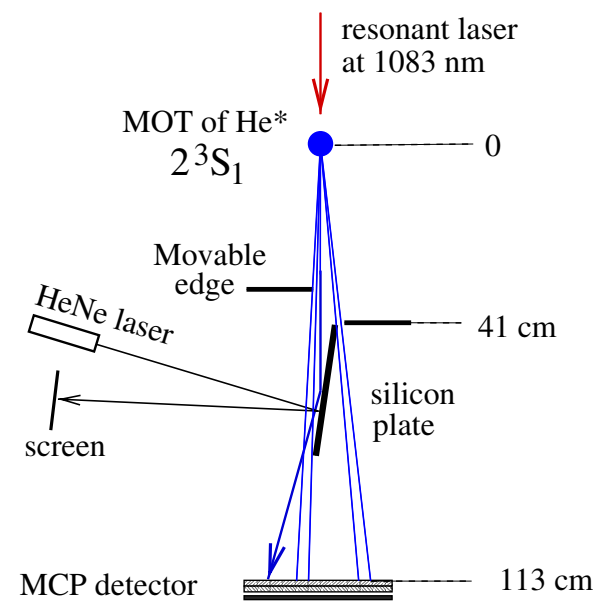

FIG. 2 (color online). Cross-sectional view of the experimental setup. described in [19]. The $\mathrm{Ne}^{*}$ atoms were released from a MOT of atoms in the $1 s_{5}\left({ }^{3} P_{2}\right)$ state. The parallel incident velocity was $v_{\|}=3 \mathrm{~m} / \mathrm{s}$, corresponding to a $0.45 \mathrm{~m}$ free fall, and the incident angle was varied between 3 and $36 \mathrm{mrad}$. The gravitational acceleration along the plate leads to a velocity spread of $0.17 \mathrm{~m} / \mathrm{s}$.

In Fig. 4(b) the same data are plotted as a function of the scaling parameter $\beta$. All data follow nearly the same curve proving the validity of the scaling law. The solid line is the estimate of the reflectivity calculated with Eq. (3). At zero incident angle it predicts the reflectivity 0.25 , instead of 1 , because we neglected multiple scattering in the derivation of the estimate. The correct behavior is recovered in the numerical simulation [22], which is plotted as a dashed line. The calculated reflectivity is larger than the measured one. Possible explanations for the deviation of experiment and theory include height variations of the ridges, the finite width of the ridges, as well as the influence of the van der Waals interaction. The calculated reflectivity represents the maximal limit that would be reached for very thin and perfectly aligned ridges.

We also analyzed the data from [19] previously measured for $\mathrm{Ne}^{*}$ on grating structures with different period $L$ and top of the ridges. These data also approximately follow the scaling law; however, we also observe a dependence on the size of the top of the ridges. The reflectivity increases generally when the width is decreased; however, a quantitative analysis is difficult because the reflectivity fluctuates with samples. The Fresnel diffraction theory is based on a thin edge, and deviations are expected, if the size of the ridge is not small compared to the distance between the ridges. The van der Waals interaction changes the effective refractive index in vicinity of the edge, and also causes additional phase shifts. We plan to analyze these effects in more detail.
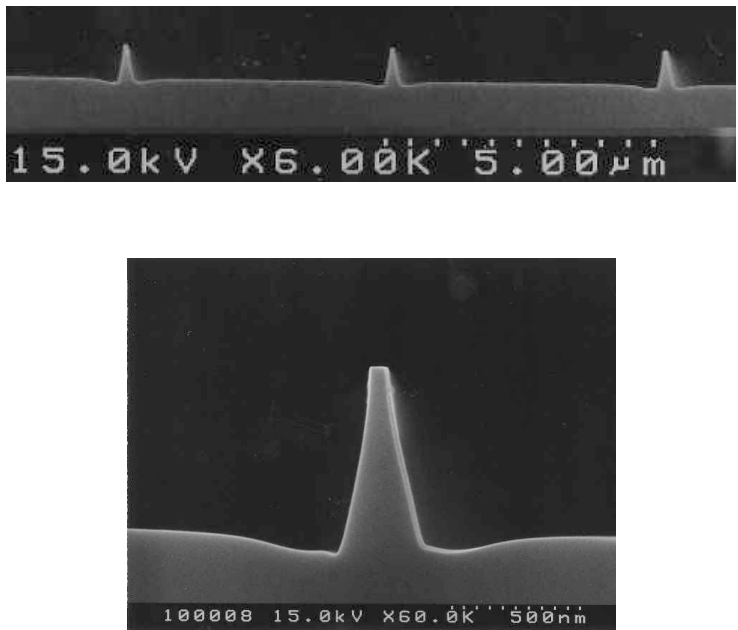

FIG. 3. Scanning microscope photograph of the silicon grating structure. Top: cross-sectional view. Bottom: expanded view of one ridge. 

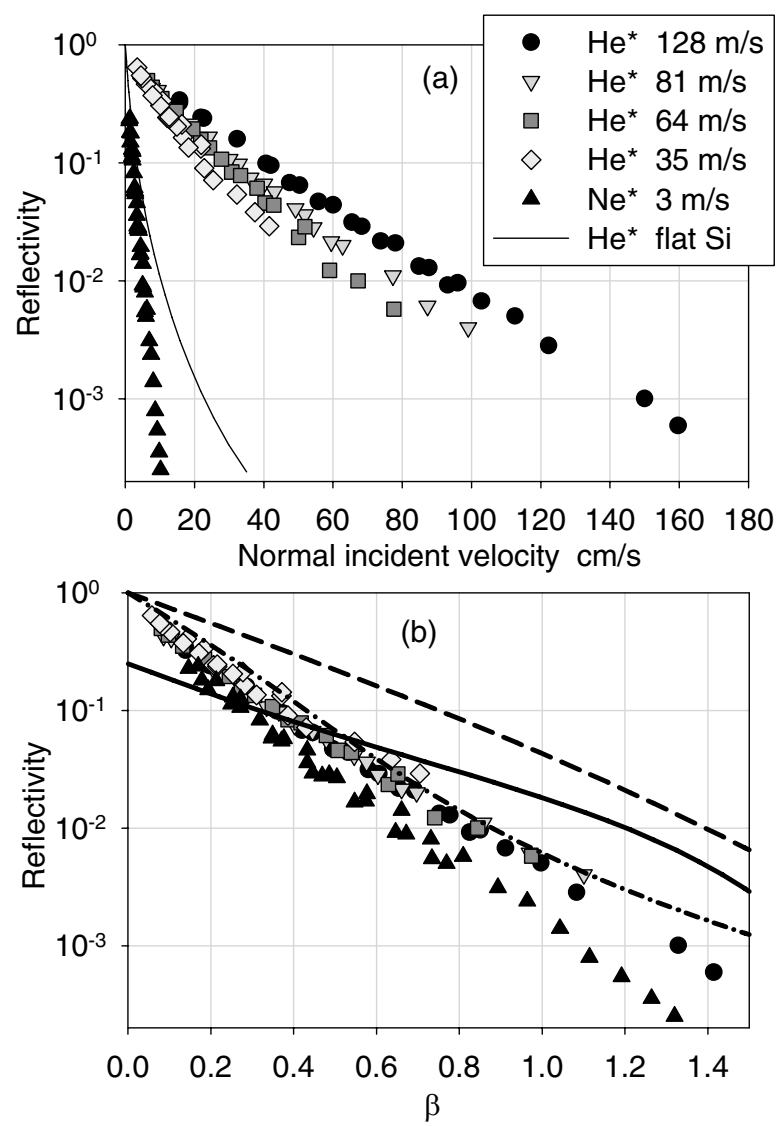

FIG. 4. (a) Reflectivity for metastable neon and helium atoms on the same ridged silicon surface. The ridges were square shaped with a period of $L=5 \mu \mathrm{m}$ and a width at the top of $100 \mathrm{~nm}$. The solid line is the reflectivity of $\mathrm{He}^{*}$ on a flat polished surface measured in [23]. (b) The reflectivity as a function of the parameter $\beta=\sqrt{L k / \pi} \theta$. The solid line is the reflectivity calculated with Eq. (3). The dashed line is the result of the numerical simulation [22], and the dash-dotted line is the empirical result obtained by assuming an equivalent medium with effective absorption rate $v_{\|} / L[22]$.

In addition, we also note that it is possible to derive an analytical estimate for the reflectivity that describes the data quite well by assuming that the array of ridges behaves like an equivalent medium with an effective absorption rate $v_{\|} / L$ [22]. This empirical result is plotted as a dash-dotted line in Fig. 4(b).

In conclusion, we have experimentally demonstrated an example of a material-independent mirror that uses Fresnel diffraction from an array of parallel ridges. For $\mathrm{He}^{*}$ and $\mathrm{Ne}^{*}$ atoms we measured a reflectivity on such a mirror that is more than 2 orders of magnitude larger than on a flat solid surface, where the reflection is material dependent.
On the ridged surface structure the reflectivity depends on the distance between the ridges and on the incident velocity of the atoms, but is insensitive to the properties of the surface material. We believe that such mirrors will be very useful to construct reflection-type phase holograms for atomic waves that combine high precision with reasonable reflectivity.

The authors thank Y. Tashiro for help in the experiment. H.O. gratefully acknowledges support from the Japan Society for the Promotion of Science. This work was partly supported by the COE Project "Coherent Optical Science" and the Grant in Aid for Scientific Research No. 16340116 of the Ministry of Education, Culture, Sports, Science and Technology, and by Matsuo Foundation, Japan.

[1] F. Shimizu, Phys. Rev. Lett. 86, 987 (2001).

[2] V. Druzhinina and M. DeKieviet, Phys. Rev. Lett. 91, 193202 (2003).

[3] T. Pasquini et al., Phys. Rev. Lett. 93, 223201 (2004).

[4] V. Nayak, D. Edwards, and N. Masuhara, Phys. Rev. Lett. 50, 990 (1983).

[5] J. Berkhout et al., Phys. Rev. Lett. 63, 1689 (1989).

[6] I. Yu et al., Phys. Rev. Lett. 71, 1589 (1993).

[7] C. Carraro and M. Cole, Prog. Surf. Sci. 57, 61 (1998).

[8] C. Henkel, C. Westbrook, and A. Aspect, J. Opt. Soc. Am. B 13, 233 (1996).

[9] R. Côté, H. Friedrich, and J. Trost, Phys. Rev. A 56, 1781 (1997).

[10] R. Doak and A. Chizmeshya, Europhys. Lett. 51, 381 (2000).

[11] A. Mody, M. Haggerty, J. Doyle, and E. Heller, Phys. Rev. B 64, 085418 (2001).

[12] H. Friedrich, G. Jacoby, and C. Meister, Phys. Rev. A 65, 032902 (2002).

[13] X. Halliwell, H. Friedrich, S. Gibson, and K. Baldwin, Opt. Commun. 224, 89 (2003).

[14] V. Balykin, V. Letokhov, Y. Ovchinnikov, and A. Sidorov, Phys. Rev. Lett. 60, 2137 (1988).

[15] M. Kasevich, D. Weiss, and S. Chu, Opt. Lett. 15, 607 (1990).

[16] C. Saba et al., Phys. Rev. Lett. 82, 468 (1999).

[17] D. Lau et al., Eur. Phys. J. D 5, 193 (1999).

[18] M. Drndić et al., Phys. Rev. A 60, 4012 (1999).

[19] F. Shimizu and J. Fujita, J. Phys. Soc. Jpn. 71, 5 (2002).

[20] F. Shimizu and J. Fujita, Phys. Rev. Lett. 88, 123201 (2002).

[21] M. Born and E. Wolf, Principles of Optics (Pergamon Press, New York, 1980), 6th ed.

[22] D. Kouznetsov and H. Oberst (to be published).

[23] H. Oberst, Y. Tashiro, K. Shimizu, and F. Shimizu (to be published). 